
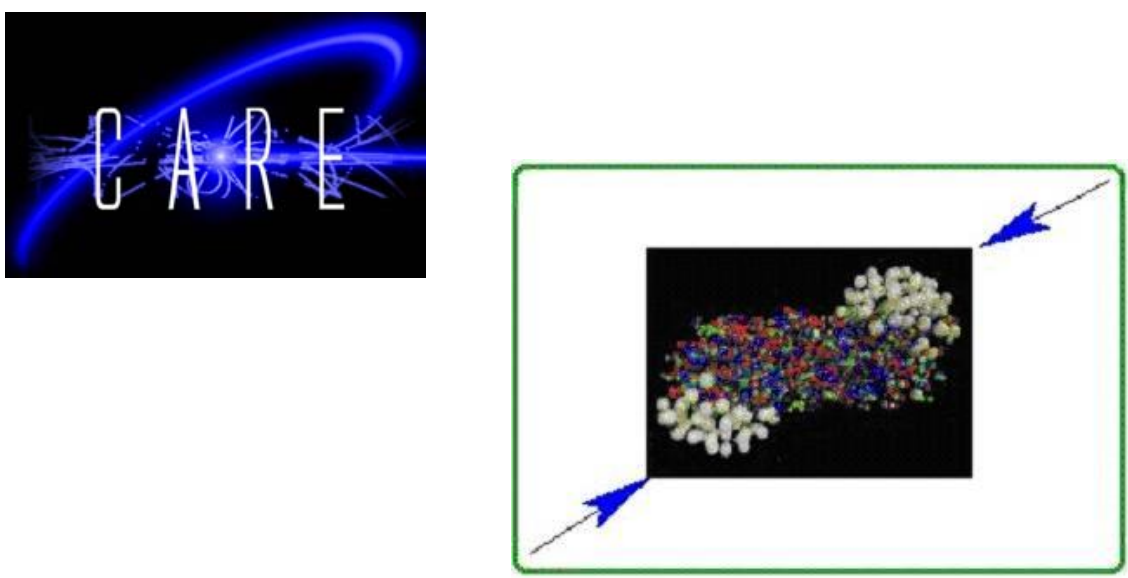

High Energy

High Intensity

Hadron Beams

\title{
LHC Upgrade Scenarios
}

\author{
F. Zimmermann
}

CERN, Geneva, Switzerland

\begin{abstract}
The EU CARE-HHH and US-LARP studies for an LHC luminosity upgrade aim at increasing the peak luminosity by a factor of 10 , to $10^{35} \mathrm{~cm}^{-2} \mathrm{~s}^{-1}$. The luminosity can be raised by rebuilding the interaction regions (IRs) in combination with a consistent change of beam parameters. In addition to advanced low-beta quadrupoles, the upgraded IRs may accommodate other new elements such as slim s.c. dipoles or quadrupoles embedded deep inside the detectors, global low-angle crab cavities, and wire compensators of long-range beam-beam effects. Important constraints on the upgrade path are the maximum acceptable number of detector pile-up events, favoring many closely spaced bunches, and the heat load on the cold-magnet beam screens, pointing towards fewer and more intense bunches. In order to translate the increased peak luminosity into a correspondingly higher integrated luminosity, the upgrade of the LHC ring should be complemented by an upgrade of the injector complex. I will present preferred upgrade scenarios for the LHC IRs including pertinent beam parameters, sketch accompanying injector enhancements, and briefly comment on a longer-term LHC energy upgrade.
\end{abstract}

Contribution to the " $22^{\text {nd }}$ Particle Accelerator Conference PAC07", Albuquerque (USA), 25-29 June 2007

Work supported by the European Community-Research Infrastructure Activity under the FP6 "Structuring the European Research Area" programme (CARE, contract number RII3-CT2003-506395) 


\title{
LHC UPGRADE SCENARIOS*
}

\author{
F. Zimmermann, CERN, Geneva, Switzerland
}

\begin{abstract}
The EU CARE-HHH and US-LARP studies for an LHC luminosity upgrade aim at increasing the peak luminosity by a factor of 10 , to $10^{35} \mathrm{~cm}^{-2} \mathrm{~s}^{-1}$. The luminosity can be raised by rebuilding the interaction regions (IRs) in combination with a consistent change of beam parameters. In addition to advanced low-beta quadrupoles, the upgraded IRs may accommodate other new elements such as slim s.c. dipoles or quadrupoles embedded deep inside the detectors, global low-angle crab cavities, and wire compensators of long-range beam-beam effects. Important constraints on the upgrade path are the maximum acceptable number of detector pile-up events, favoring many closely spaced bunches, and the heat load on the cold-magnet beam screens, pointing towards fewer and more intense bunches. In order to translate the increased peak luminosity into a correspondingly higher integrated luminosity, the upgrade of the LHC ring should be complemented by an upgrade of the injector complex. I will present preferred upgrade scenarios for the LHC IRs including pertinent beam parameters, sketch accompanying injector enhancements, and briefly comment on a longer-term LHC energy upgrade.
\end{abstract}

\section{INTRODUCTION}

The Large Hadron Collider (LHC) now under construction at CERN will collide two proton beams with a centreof-mass energy of $14 \mathrm{TeV}$ (7 times the energy of the Tevatron's proton-antiproton collisions) at design and ultimate luminosities of $10^{34} \mathrm{~cm}^{-2} \mathrm{~s}^{-1}$ and $2.3 \times 10^{34} \mathrm{~cm}^{-2} \mathrm{~s}^{-1}$ (about 100 times that of the Tevatron). LHC beam commissioning will commence in 2008. The LHC proton beams will circulate in separate pipes and cross each other at the four detectors of the two high-luminosity experiments ATLAS and CMS, the B physics experiment LHC-B, and the ion-collision experiment ALICE, respectively.

Possible LHC upgrade paths, first looked at around 2001 [1], are being further developed by the CARE [2] HHH network [3]. In parallel the CARE NED activity [4] has worked towards higher field magnets (potentially useful for an eventual LHC energy upgrade). Both HHH and NED studies proceed in collaboration with US LARP [5]. The goal of the LHC upgrade studies is to raise the luminosity ten times to $10^{35} \mathrm{~cm}^{-2} \mathrm{~s}^{-1}$. Presently, a staged upgrade is being considered, where in a first phase the inner triplets of the LHC high-luminosity interaction regions will be replaced by about 2012 .

The original baseline scheme for the LHC upgrade [1] foresaw operating at the ultimate bunch intensity of $1.7 \times$

\footnotetext{
${ }^{*}$ We acknowledge the support of the European Community-Research Infrastructure Initiative under the FP6 "Structuring the European Research Area" programme (CARE, contract number RII3-CT-2003-506395).
}

$10^{11}$, with twice the number of bunches, i.e., $12.5 \mathrm{~ns}$ bunch spacing, a crossing angle about 50\% larger than nominal, and correspondingly bunches of half the nominal length. The bunches were supposed to be shortened through a combination of higher harmonic rf and reduced longitudinal emittance. The attractiveness of this scenario was that it could achieve a luminosity of $10^{35} \mathrm{~cm}^{-2} \mathrm{~s}^{-1}$, ten times higher than nominal, with less than 100 events per crossing (about 20 for the nominal LHC). As a backup long superbunches were also considered, but later these proved not to be useful for the physics experiments. More recently, the short-bunch baseline scheme had to be given up as well, since the expected heat load from image currents and synchrotron radiation alone, without including any contribution from electron cloud, reaches the maximum cooling capacity of $2.4 \mathrm{~W} / \mathrm{m}$ per beam, ultimately limited by the hydraulic impedance of the beam-screen capillaries [6]. Towards the end of 2006 two new upgrade scenarios have emerged [7], which compromise between the tolerable arc heat load and an acceptable number of pile-up events.

In this paper, I describe these two new alternative scenarios for the LHC upgrade, together with the pertinent beam parameters, interaction-region layouts, hardware requirements, merits and challenges. I also explore the luminosity evolution for the two cases, possible approaches for luminosity leveling, and the compatibility with a Super-LHCb. The planned upgrade of the LHC injector complex and a future energy upgrade are briefly mentioned.

\section{TWO SCENARIOS}

When operating at the beam-beam limit with alternating planes of crossing at two interaction points (IPs), the luminosity can be expressed as [8]

$$
L \approx \frac{f_{\text {rev }} \gamma}{2 r_{p}} n_{b} \frac{1}{\beta^{*}} N_{b} \Delta Q_{b b} F_{\text {profile }} F_{\mathrm{hg}},
$$

where $\Delta Q_{b b}$ denotes the total beam-beam tune shift, limited to about 0.01 according to experience at previous hadron colliders, $f_{\text {rev }}$ the revolution frequency, $F_{\text {profile }}$ a form factor depending on the longitudinal profile (equal to 1 for a Gaussian and $\sqrt{2}$ for a uniform profile) and $F_{\mathrm{hg}}$ the reduction factor due to the hourglass effect, which is relevant for bunch lengths comparable to, or smaller than, the IP beta function. Collision of round beams is assumed. Other variables are defined in Table 1, which compares the parameters of the nominal and ultimate LHC, with those for the old baseline, and the two new upgrade scenarios. The parameters in (1) which differ from the ultimate LHC configuration are $\beta^{*}, N_{b}, \Delta Q_{b b}, F_{\text {profile }}$ and $n_{b}$ in one of these scenarios, and $\beta^{*}, \Delta Q_{b b}$ and $F_{\mathrm{hg}}$ in the other.

In the "early-separation" (ES) scenario [9] one stays with the ultimate LHC beam, squeezes $\beta^{*}$ down to about 
$10 \mathrm{~cm}$ in ATLAS and CMS; adds early-separation dipoles inside the detectors starting at about $3 \mathrm{~m}$ from the IP; possibly also includes a quadrupole doublet at about 13 $m$ from the IP [10]; and uses crab cavities [11] so that the effective Piwinski angle becomes zero. This scenario implies installation of new hardware inside the ATLAS and CMS detectors, as well as the first ever hadron beam crab cavities. The latter gain a factor 2 to 5 in luminosity [9]. The maximum bunch intensity $N_{b}$ is linked to the limit on the total beam-beam tune shift for two IPs, via $\left|\Delta Q_{b b}\right|=N_{b} r_{p} \beta^{*} /\left(2 \pi \gamma \sigma^{* 2}\right)$, where $\sigma^{*}$ is the transverse rms beam size at the IP. A corresponding IR layout is sketched in Fig. 1. Its merits are the negligible effect of most long-range collisions thanks to the early separation, the absence of any geometric luminosity loss except for the hourglass effect, and no increase in the beam current beyond ultimate. Challenges include the early separation dipoles 'D0' deep inside the detector, an optional s.c. quadrupole doublet 'Q0' also embedded, strong largeraperture low- $\beta$ quadrupoles based on $\mathrm{Nb}_{3} \mathrm{Sn}$, use of crab cavity for hadron beams [11], the remaining 4 parasitic collisions at 4-5 $\sigma$ separation, a significant off-momentum beta beating which may degrade collimation efficiency, plus low beam and luminosity lifetimes (proportional to $\beta^{*}$ ).

In the "large Piwinski angle" (LPA) scenario the bunch spacing is doubled, to $50 \mathrm{~ns}$; longer, longitudinally flat, and more intense bunches are collided with a large Piwinski angle of $\phi \equiv \theta_{c} \sigma_{z} /\left(2 \sigma^{*}\right) \approx 2$; the IP beta function is reduced by a more moderate factor of 2 to $\beta^{*} \approx 25 \mathrm{~cm}$; and longrange beam-beam wire compensators [12] are installed upstream of the inner triplets. This regime of large $\phi$ and uniform bunch profile allows raising the bunch intensity $N_{b}$ in (1) and thereby the luminosity, as lengthening the bunches in proportion to $N_{b}$ maintains a constant value of $\Delta Q_{b b}$. Figure 2 illustrates the IR layout for this upgrade option. Its merits are the absence of accelerator elements inside the detector, no crab cavities, reduced IR chromaticity, and relaxed IR quadrupoles (for $\beta^{*} \approx 25 \mathrm{~cm}$ various optics solutions based on large-aperture NbTi quadrupoles exist [13]). Challenges are the operation with large Piwinski angle, unproven for hadron beams, the high bunch charge, in particular the beam production and acceleration through the SPS, the larger beam current, and, lastly, the wire compensation (almost established).

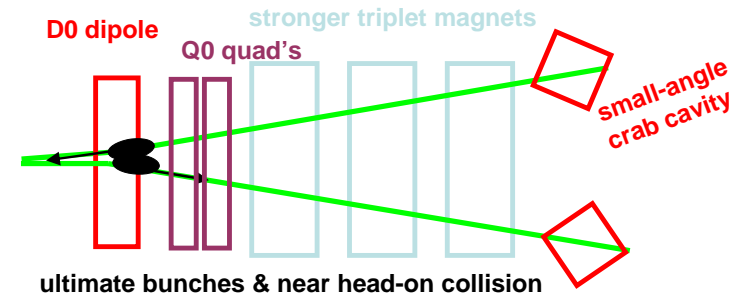

Figure 1: Interaction-region layout for the early-separation (ES) scheme, with highly squeezed optics $\left(\beta^{*} \approx 8 \mathrm{~cm}\right)$.

Figure 3 compares the luminosity evolution for the two scenarios. A turn-around time (the time between the end of a collision run and the start of the next collisions) of

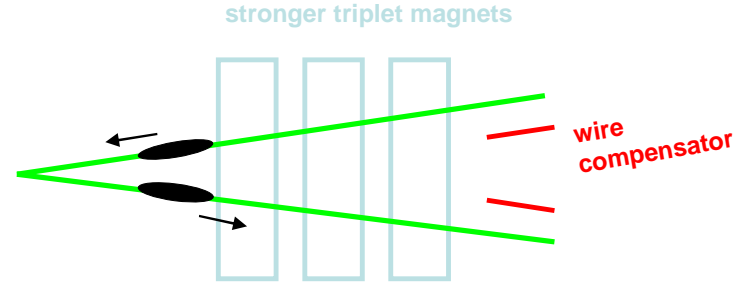

long bunches \& nonzero crossing angle \& wire compensation

Figure 2: Interaction-region layout for large-Piwinskiangle (LPA) upgrade with an IP beta function of $0.25 \mathrm{~m}$.

$5 \mathrm{~h}$ and the corresponding optimum run durations from Table 1 are assumed. The dashed lines indicate the respective time-averaged luminosities. The instantaneous luminosity decays as $L(t)=\hat{L} /\left(1+t / \tau_{\text {eff }}\right)^{2}$ with $\tau_{\text {eff }} \equiv$ $n_{b} N_{b}(0) /\left(\hat{L} \sigma_{\text {tot }} n_{I P}\right)$ the effective beam lifetime due to burn-off at the collision points, $\sigma_{\text {tot }} \approx 100 \mathrm{mb}$ the relevant total cross section, $n_{I P}$ the number of IPs, and $\hat{L}$ the initial peak luminosity. The optimum average luminosity is

$$
L_{\mathrm{ac}}=\hat{L} \frac{\tau_{\mathrm{eff}}}{\left(\tau_{\mathrm{eff}}^{1 / 2}+T_{\mathrm{ta}}^{1 / 2}\right)^{2}},
$$

where $T_{t a}$ denotes the turn-around time. The optimum run time $T_{\text {run }}$ is the geometric mean of effective lifetime and turn-around time: $T_{\text {run }}=\sqrt{\tau_{\text {eff }} T_{\mathrm{ta}}}$.

In Fig. 3 it can be seen that the luminosity for the ES scenario starts higher, but decays faster than for the LPA case, leading to shorter runs. The average luminosity values are nearly identical. The high initial peak luminosity for ES may not be useful for physics in view of possibly required set-up and tuning periods. On the other hand, Fig. 4 shows that the average event pile up for the ES option is about $30-40 \%$ lower than that for the LPA case.

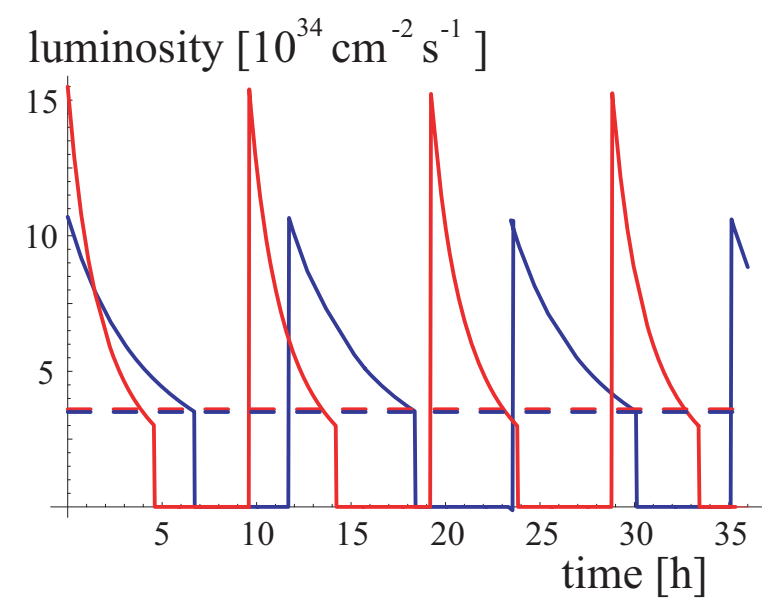

Figure 3: Ideal luminosity evolution for the ES (red) and LPA scenarios (blue), considering the optimum run duration for a turn-around time of $5 \mathrm{~h}$. The dashed lines indicate the corresponding time-averaged luminosities.

Either upgrade scenario could potentially be adapted for crab-waist collisions, i.e., collisions where the waist position of the vertical beta function $\beta_{y}$ depends on a particle's horizontal location, as $s=-x / \theta_{c}$ [14]. Such $x$-dependent 
Table 1: Parameters for the (1) nominal and (2) ultimate LHC compared with those for three upgrade scenarios with (3) shorter bunches at 12.5-ns spacing [old baseline], (4) more strongly focused ultimate bunches with early separation at 25-ns spacing [ES], (5) longer intense flat bunches at 50-ns spacing in a regime of large Piwinski angle [LPA]. The numbers refer to the performance without luminosity leveling.

\begin{tabular}{|c|c|c|c|c|c|c|}
\hline parameter & symbol & nominal & ultimate & old & $\mathrm{ES}$ & $\overline{\mathrm{LPA}}$ \\
\hline number of bunches & $\overline{n_{b}}$ & 2808 & 2808 & 5616 & 2808 & 1404 \\
\hline protons per bunch & $N_{b}\left[10^{11}\right]$ & 1.15 & 1.7 & 1.7 & 1.7 & 4.9 \\
\hline bunch spacing & $\Delta t_{\mathrm{sep}}[\mathrm{ns}]$ & 25 & 25 & 12.5 & 25 & 50 \\
\hline average current & $I[\mathrm{~A}]$ & 0.58 & 0.86 & 1.72 & 0.86 & 1.22 \\
\hline normalized transverse emittance & $\gamma \epsilon[\mu \mathrm{m}]$ & 3.75 & 3.75 & 3.75 & 3.75 & 3.75 \\
\hline longitudinal profile & & Gaussian & Gaussian & Gaussian & Gaussian & uniform \\
\hline rms bunch length & $\sigma_{z}[\mathrm{~cm}]$ & 7.55 & 7.55 & 3.78 & 7.55 & 11.8 \\
\hline beta function at IP1 $\& 5$ & $\beta^{*}[\mathrm{~m}]$ & 0.55 & 0.5 & 0.25 & 0.08 & 0.25 \\
\hline (effective) crossing angle & $\theta_{c}[\mu \mathrm{rad}]$ & 285 & 315 & 445 & 0 & 381 \\
\hline Piwinski angle & $\phi$ & 0.4 & 0.75 & 0.75 & 0 & 2.01 \\
\hline hourglass factor & $F_{\mathrm{hg}}$ & 1.00 & 1.00 & 1.00 & 0.86 & 0.99 \\
\hline peak luminosity & $\hat{L}\left[10^{34} \mathrm{~cm}^{-2} \mathrm{~s}^{-1}\right]$ & 1.0 & 2.3 & 9.2 & 15.5 & 10.6 \\
\hline events per crossing & & 19 & 44 & 88 & 294 & 403 \\
\hline rms length of luminous region & $\sigma_{\text {lum }}[\mathrm{mm}]$ & 45 & 43 & 21 & 53 & 37 \\
\hline initial luminosity lifetime & $\tau_{\mathrm{L}}[\mathrm{h}]$ & 22.2 & 14.3 & 7.2 & 2.2 & 4.5 \\
\hline average luminosity $\left(T_{\mathrm{ta}}=10 \mathrm{~h}\right)$ & $L_{\mathrm{av}}\left[10^{34} \mathrm{~cm}^{-2} \mathrm{~s}^{-1}\right]$ & 0.5 & 0.9 & 2.7 & 2.4 & 2.5 \\
\hline optimum run time $\left(T_{\mathrm{ta}}=10 \mathrm{~h}\right)$ & $T_{\text {run }}[\mathrm{h}]$ & 21.2 & 17.0 & 12.0 & 6.6 & 9.5 \\
\hline average luminosity $\left(T_{\mathrm{ta}}=5 \mathrm{~h}\right)$ & $L_{\mathrm{av}}\left[10^{34} \mathrm{~cm}^{-2} \mathrm{~s}^{-1}\right]$ & 0.6 & 1.2 & 3.7 & 3.6 & 3.5 \\
\hline optimum run time $\left(T_{\mathrm{ta}}=5 \mathrm{~h}\right)$ & $T_{\text {run }}[\mathrm{h}]$ & 15.0 & 12.0 & 8.5 & 4.6 & 6.7 \\
\hline e-cloud heat load for $\delta_{\max }=1.4$ & $P_{\mathrm{ec}}[\mathrm{W} / \mathrm{m}]$ & 1.07 & 1.04 & 13.3 & 1.0 & 0.4 \\
\hline e-cloud heat load for $\delta_{\max }=1.3$ & $P_{\mathrm{ec}}[\mathrm{W} / \mathrm{m}]$ & 0.44 & 0.6 & 7.9 & 0.6 & 0.1 \\
\hline SR heat load & $P_{\mathrm{SR}}[\mathrm{W} / \mathrm{m}]$ & 0.17 & 0.25 & 0.5 & 0.25 & 0.36 \\
\hline image-current heat load & $P_{\text {ic }}[\mathrm{W} / \mathrm{m}]$ & 0.15 & 0.33 & 1.85 & 0.33 & 0.70 \\
\hline
\end{tabular}

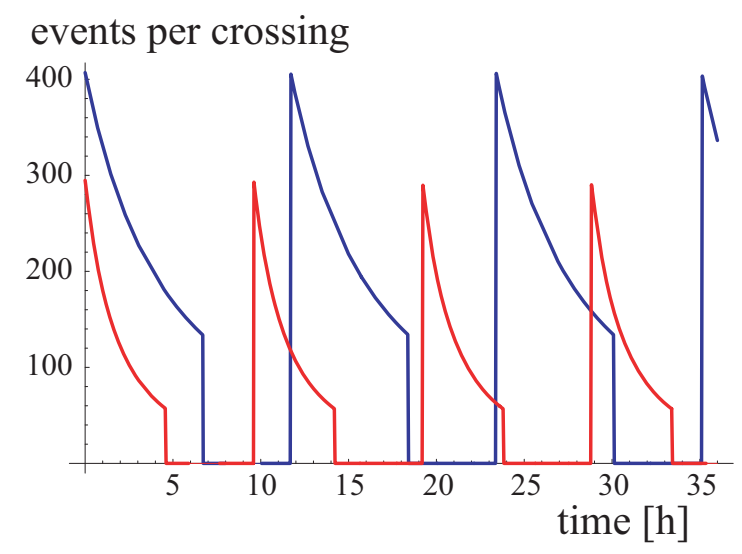

Figure 4: Number of events per crossing as a function of time for the same two upgrade scenarios and luminosity time evolutions as in Fig. 3.

waist shifts can be realized by adding sextupoles with the right horizontal and vertical betatron phase advances from the IP. To this end it may be preferable to collide flat beams with $\beta_{x} \gg \beta_{y}$ [15], which also makes optimum use of the available aperture in the low-beta quadrupoles.

\section{LUMINOSITY LEVELING}

The LHC experiments would prefer a more constant luminosity, i.e., less pile up at the start of a run, and higher luminosity at the end. This luminosity leveling could be achieved either by dynamic $\beta^{*}$ squeeze or crossing angle changes [16] or changes in the crab-cavity rf voltage for $\mathrm{ES}$, and equally by dynamic $\beta *$ squeeze or via bunchlength reduction for LPA.

Figure 5 illustrates the typical range of $\beta^{*}$ and total bunch length $l_{b}$ in case of luminosity leveling for the LPA upgrade. The various curves refer to different constant rates of events per crossing. Since with leveling the luminosity is held constant, equal to $L_{0}$, the beam intensity decreases linearly with time $t$ as $N_{b}=N_{b 0}-L_{0} \sigma_{\text {tot }} n_{I P} / n_{b} t$. The accessible intensity range $\Delta N_{b \text {, max }}$ is limited, e.g., by the range of the leveling variable, for example by the minimum value of $\beta^{*}$. Then the length of a run is $T_{\text {run }}=\Delta N_{b, \max } n_{b} /\left(L_{0} \sigma_{\text {tot }} n_{I P}\right)$, and the average luminosity with leveling becomes

$$
L_{\mathrm{av}, \mathrm{lev}}=L_{0} /\left(1+\frac{\Delta N_{b, \max } n_{b}}{L_{0} \sigma_{\mathrm{tot}} n_{I P}} T_{t a}\right) .
$$

Table 2 compares event rates, run times, and average luminosity values which can be achieved in ES and LPA. Figure 6 illustrates the luminosity evolution with leveling, and Fig. 7 compares the variation of the total beam-beam tune shift with bunch intensity for leveling via $\beta^{*}$, via effective crossing angle $\theta_{c}$, or via the bunch length. In case of $\beta^{*}$ variation, the tune shift decreases during the store. By contrast, when leveling via the bunch length or crossing angle the tune shift increases. Figures 8 and 9 display the average 
Table 2: Event rate, run time, and average luminosity for the two upgrade scenarios with luminosity leveling.

\begin{tabular}{|lcc|}
\hline & ES & LPA \\
\hline events/crossing & 300 & 300 \\
optimum run time & N/A & $2.5 \mathrm{~h}$ \\
av. luminosity $\left[10^{34} \mathrm{~cm}^{-2} \mathrm{~s}^{-1}\right]$ & N/A & 2.6 \\
\hline events/crossing & 150 & 150 \\
optimum run time & $2.5 \mathrm{~h}$ & $14.8 \mathrm{~h}$ \\
av. luminosity $\left[10^{34} \mathrm{~cm}^{-2} \mathrm{~s}^{-1}\right]$ & 2.6 & 2.9 \\
\hline events/crossing & 75 & 75 \\
optimum run time & $9.9 \mathrm{~h}$ & $26.4 \mathrm{~h}$ \\
av. luminosity $\left[10^{34} \mathrm{~cm}^{-2} \mathrm{~s}^{-1}\right]$ & 2.6 & 1.7 \\
\hline
\end{tabular}

luminosity and the optimum run time as a function of the minimum $\beta^{*}$ at the end of the dynamic squeeze, for both ES and LPA. These figures illustrate the sensitivity of the average luminosity to the $\beta^{*}$ reach of the IR optics.
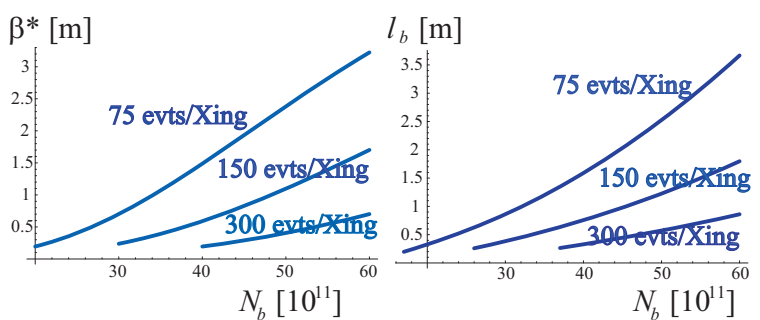

Figure 5: IP beta function (left) and full bunch length (right) as a function of bunch intensity for luminosity leveling in the LPA scenario. The number of events per crossing is held constant for each curve, with values as indicated.

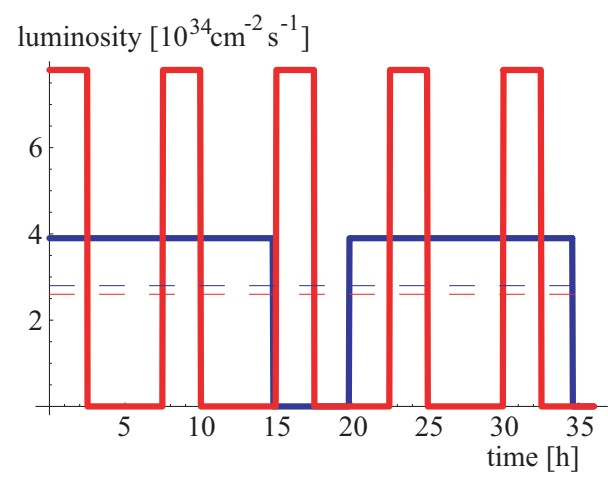

Figure 6: Luminosity evolution for the ES (red) and LPA scenarios (blue) with leveling at a constant rate of 150 events/crossing, and the optimum run duration for a turnaround time of $5 \mathrm{~h}$. The dashed lines indicate the corresponding time-averaged luminosities.

\section{BUNCH STRUCTURE \& LHCB}

The bunch patterns for various LHC configurations are compared in Fig. 10.

An upgrade of $\mathrm{LHCb}$ to Super-LHCb is planned, in order

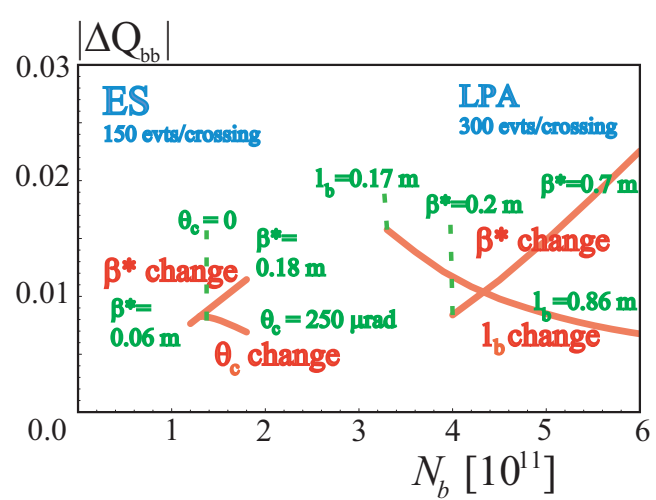

Figure 7: Example tune shifts with luminosity leveling.

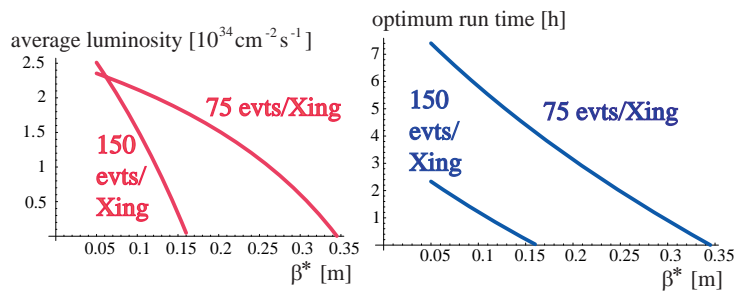

Figure 8: Average luminosity (left) and run time (right) vs. final $\beta^{*}$ for ES with $\beta^{*}$ leveling.

to handle luminosities of up to $2 \times 10^{33} \mathrm{~cm}^{-2} \mathrm{~s}^{-1}$. Due to its asymmetric location in the ring, $\mathrm{LHCb}$ will see no collisions for a beam with 50-ns spacing colliding in IP1 and 5 (ATLAS and CMS).

In the case of LPA with 50-ns spacing, satellite bunches can be added in between the main bunches. Such satellites may be produced by asymmetric bunch splitting in the PS (possibly large fluctuation). In LHCb these satellites collide with main bunches at $25-n$ s time intervals. The intensity of the satellites should be lower than $3 \times 10^{10}$ protons per bunch (1/16th of the main bunch charge) in order to add less than $5 \%$ to the total tune shift and also to avoid electron-cloud problems. A beta function of about $3 \mathrm{~m}$ would result in the desired luminosity equivalent to $2 \times 10^{33} \mathrm{~cm}^{2} \mathrm{~s}^{-1}$. This value of $\beta^{*}$ is easily possible with the present LHCb IR magnets and layout.

For the ES scenario with 25-ns bunch spacing, head-on collisions at Super-LHCb would contribute to the beambeam tune shift of the bunches colliding in ATLAS and CMS. Two ways out are (1) colliding only during the second half of each store when the beam-beam tune shifts from IP1 and 5 have sufficiently decreased below the beam-beam
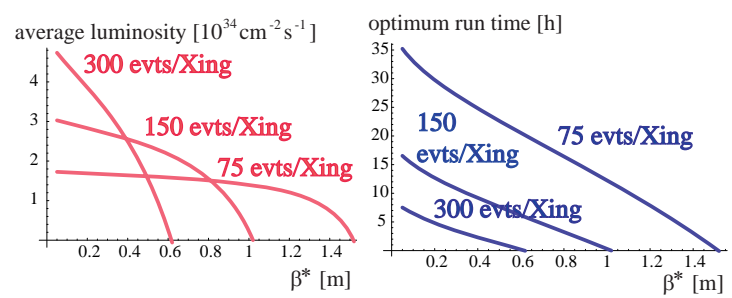

Figure 9: Average luminosity (left) and run time (right) vs. final $\beta^{*}$ for LPA with $\beta^{*}$ leveling. 
limit, and (2) introducing a transverse collision offset, albeit the latter raises concerns about offset stability, interference with collimation, poor beam lifetime, background etc. Requiring an $\mathrm{LHCb}$ contribution to the total tune shift of less than $10 \%$ implies transverse beam-beam offsets larger than $4.5 \sigma$, and $\beta^{*} \approx 8 \mathrm{~cm}$, which is incompatible with the present LHCb IR configuration. For either ES option, the average luminosity delivered to Super-LHCb is considerably lower than for the LPA case with satellites.

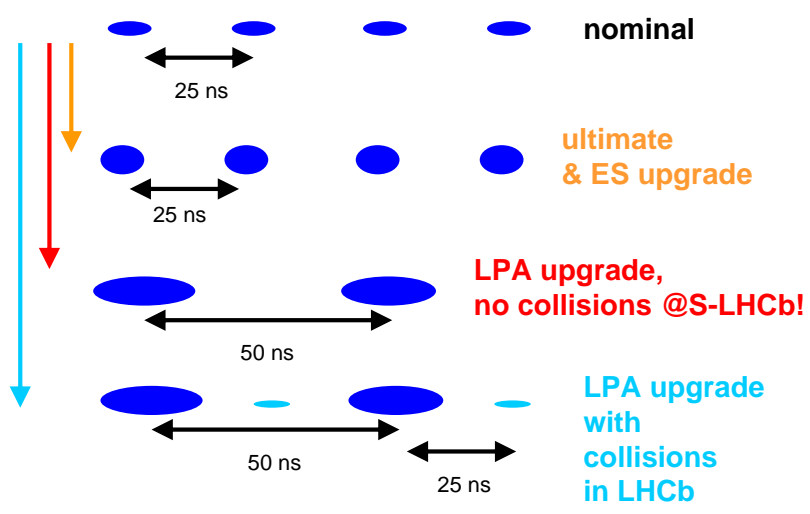

Figure 10: Bunch structures for nominal LHC, ultimate, ES upgrade, LPA upgrade, and LPA with satellite-bunch collisions at Super-LHCb.

\section{INJECTOR AND ENERGY UPGRADE}

An upgrade of the LHC injector complex is needed for producing the ultimate beam. In addition to the improved brightness, the planned injector upgrade is also likely to reduce the LHC turn-around time, and thereby to increase the average luminosity. A new injector linac will first raise the injection energy into the PS Booster to $160 \mathrm{MeV}$ and, in its final 5-GeV version, render the booster obsolete [17]. The PS itself will be replaced by a larger-circumference ring, "PS2", with a higher injection energy of $5 \mathrm{GeV}$ [17]. The PS2 must be complemented by SPS enhancements, and by new transfer lines. For the ES scenario, the beam production in the injector complex could remain the same as for the ultimate LHC beam. For the LPA scenario, several methods of beam production are conceivable. For example, omitting the last bunch splitting in the PS (or future PS2), bunches of up to $5.0 \times 10^{11}$ protons spaced by 50 ns could directly be injected into the SPS, where a larger than nominal longitudinal emittance would help maintaining beam stability. and possibly imply upgrades of the SPS and LHC rf systems. At least three different techniques for flattening the bunches longitudinally are available [18, 19].

A strong physics case calls for higher beam energies. The LHC beam energy is determined by the main dipole field, whose nominal value of $8.39 \mathrm{~T}$ corresponds to $7 \mathrm{TeV}$. At LBNL a proof-of-principle magnet based on $\mathrm{Nb}_{3} \mathrm{Sn}$ s.c. material reached $16 \mathrm{~T}$ with a $10-\mathrm{mm}$ aperture [20]. NED [4] aimed at developing a large-aperture (up to 88 $\mathrm{mm}), 15-\mathrm{T}$ dipole-magnet model. A 24-T block-coil dipole for an LHC energy tripler is being developed by Texas
A\&M University [21]. It employs high- $T_{c}$ superconductor (Bi-2212) in the inner high-field windings and $\mathrm{Nb}_{3} \mathrm{Sn}$ for the outer low-field windings.

\section{SUMMARY}

We have described two scenarios reaching an LHC peak luminosity above $10^{35} \mathrm{~cm}^{-2} \mathrm{~s}^{-1}$ for which both heat load and the number of events per crossing remain acceptable. The early-separation scenario pushes $\beta^{*}$. It requires slim s.c. magnets inside the detector, crab cavities, and most likely $\mathrm{Nb}_{3} \mathrm{Sn}$ quadrupoles. This scheme is attractive if the total beam current in LHC is limited. Luminosity leveling is possible in this case by means of varying the crossing angle or $\beta^{*}$. The large-Piwinski-angle scenario features fewer and longer bunches of higher charge. It can be realized with NbTi technology if needed and it is compatible with LHCb. Open issues are the SPS and beam-beam effects in this parameter regime. Here, the luminosity leveling may be done via the bunch length and/or also via $\beta^{*}$.

The first two years of LHC operation will clarify the severity of electron cloud, long-range beam-beam collisions, impedance etc. At the same time, the first physics results will indicate whether or not magnetic elements can be installed inside the detectors. These two experiences are likely to decide the upgrade path, which might eventually combine aspects of the two scenarios. Until then both upgrade options are kept open.

\section{ACKNOWLEDGEMENTS}

Many colleagues from CERN and around the world contributed to the ideas presented in this paper. In particular, I thank W. Scandale and J.-P. Koutchouk for numerous helpful discussions. This paper is dedicated to the memory of F. Ruggiero (1957-2007), who initiated and guided the LHC accelerator upgrade studies.

\section{REFERENCES}

[1] O. Brüning et al, LHC-PROJECT-Report-626 (2002).

[2] Site http://esgard.lal.in2p3.fr/Project/Activities/Current/

[3] Site http://care-hhh.web.cern.ch/CARE-HHH/

[4] Site http://lt.tnw.utwente.nl/project.php?projectid=9

[5] Site http://www.agsrhichome.bnl.gov/LARP/

[6] L. Tavian, "Cryogenic Limits," in Ref. [7].

[7] HHH LUMI'06 workshop, Valencia, CERN-2007-002; http://care-hhh.web.cern.ch/CARE-HHH/LUMI-06/

[8] F. Ruggiero, F. Zimmermann, PRST-AB 5, 061001 (2002).

[9] E. Todesco et al, THPAN072, this conference.

[10] E. Laface et al, THPAN067, this conference.

[11] R. Calaga et al, TUPAS089, this conference.

[12] U. Dorda et al, TUPAN091, this conference.

[13] O. Brüning et al., LHC-PROJECT-Report-1008 (2007).

[14] P. Raimondi, MOZAKI02, this conference.

[15] S. Fartoukh, LHC MAC, 16.06.2006.

[16] G. Sterbini et al, LHC-Project-Note-403 (2007).

[17] R. Garoby, Proc. LUMI'06, Valencia, CERN-2007-002.

[18] A. Blas et al, EPAC2000, Vienna, 1528 (2000)

[19] C. Carli, M. Chanel, HB2002 Batavia, AIP CP 642 (2002).

[20] LBNL S.c. Magnet Program Newsletter, no. 2 (2003).

[21] P. McIntyre, A. Sattorov, PAC'05, Knoxville (2005). 